\title{
Intensity oscillations and heating of the coronal X-ray bright points from Hinode/XRT
}

\author{
R. Kariyappa and B. A. Varghese
}

Indian Institute of Astrophysics, Bangalore 560 034, India

e-mail: rkari@iiap.res.in

Received 23 November 2007 / Accepted 16 April 2008

\section{ABSTRACT}

\begin{abstract}
Aims. Our aim is to investigate the intensity oscillations in coronal X-ray Bright Points (XBPs).
Methods. We analysed a $7 \mathrm{~h}$ long time sequence of the soft X-ray images obtained on April 14, 2007 with a 2 min cadence using the X-Ray Telescope (XRT) on-board the Hinode mission. We use SSW in IDL to derive the time series of 14 XBPs and 2 background regions. For the first time, we have tried to use power spectrum analysis on XBP data to determine the periods of intensity oscillations. Results. The power spectra of XBPs show several significant peaks at different frequencies corresponding to time scales that range from a few minutes to hours. The light curves of all the XBPs give the impression that the XBPs can be grouped into three classes depending on emission levels: (i) weak XBPs; (ii) bright XBPs; and (iii) very strong XBPs. The periods of intensity oscillation are consistent in all the XBPs and are independent of their brightness level, suggesting that the heating mechanisms in all the three groups of XBPs are similar. The different classes of XBPs may be related to the different strengths of the magnetic field with which they have been associated.
\end{abstract}

Key words. Sun: corona - Sun: magnetic fields - Sun: oscillations - Sun: X-rays, gamma rays - Sun: atmosphere

\section{Introduction}

Solar coronal X-ray bright points (XBPs) have been an enigma since their discovery in late 1960' (Vaiana et al. 1970). XBPs have been studied in great detail using Skylab and Yohkoh X-ray images (Golub et al. 1974; Harvey 1996; Nakakubo \& Hara 1999; Longcope et al. 2001; Hara \& Nakakubo 2003). Their correspondence with small bipolar magnetic regions was discovered by combining ground-based magnetic field measurements with simultaneous space-born X-ray imaging observations (Krieger et al. 1971; Golub et al. 1977). The number of XBPs (daily) found on the Sun varies from several hundreds up to a few thousands (Golub et al. 1974). Zhang et al. (2001) found a density of 800 XBPs for the entire solar surface at any given time. It is known that the observed XBP number is anti-correlated with the solar cycle, but this is an observational bias and the number density of XBPs is nearly independent of the 11-yr solar activity cycle (Nakakubo \& Hara 1999; Sattarov et al. 2002; Hara \& Nakakubo 2003). Golub et al. (1974) found that the diameters of the XBPs are around 10-20 arcsec and their life times range from 2 h to 2 days (Zhang et al. 2001). Studies have indicated the temperatures to be fairly low, $T=2 \times 10^{6} \mathrm{~K}$, and electron densities $n_{\mathrm{e}}=5 \times 10^{9} \mathrm{~cm}^{-3}$ (Golub \& Pasachoff 1997), although cooler XBPs exist (Habbal 1990). XBPs are also useful as tracers of coronal rotation (Kariyappa 2008) and contribute to the Solar X-ray irradiance variability (DeLuca \& Saar 2008; Kariyappa \& DeLuca 2008). Assuming that almost all XBPs represent new magnetic flux emerging at the solar surface, their overall contribution to the solar magnetic flux would exceed that of the active regions (Golub \& Pasachoff 1997). Since a statistical interaction of the magnetic field is associated with the production of XBPs, the variation of the XBP number on the Sun will be a measure of the magnetic activity of its origin.
Bright points are also observed in the chromosphere using high resolution $\mathrm{CaII} \mathrm{H}$ and $\mathrm{K}$ spectroheliograms and filtergrams. Extensive studies have been conducted to determine their dynamical evolution, the contribution to chromospheric oscillations and heating, and to UV irradiance variability (e.g. Liu 1974; Cram \& Damé 1983; Kariyappa et al. 1994; Kariyappa 1994 \& 1996; Kariyappa \& Pap 1996; Kariyappa 1999; Kariyappa et al. 2005). The oscillations of the bright points at the higher chromosphere have been investigated using SOHO/SUMER Lyman series observations (Curdt \& Heinzel 1998; Kariyappa et al. 2001). It is known from these studies that the chromospheric bright points are associated with 3 min periods in their intensity variations, using power spectra analysis.

Oscillations have been investigated in XBPs using Yohkoh/SXT (Strong et al. 1992) and intensity variations on time scales of a few minutes to hours have been found. In 2002, we investigated the intensity oscillations at the sites of XBPs using Yohkoh/SXT observations. We found that there were several long gaps in the time sequence data and hence it was difficult to derive the power spectra precisely. The studies related to intensity oscillations of the XBPs and their contribution to coronal heating were not well discussed with Yohkoh/SXT observations. The X-ray Telescope on Hinode, XRT, has made long and continuous high temporal and spatial resolution time sequence observations of XBPs. In addition the angular resolution of XRT is $1^{\prime \prime}$, which is almost three times better than that of Yohkoh/SXT instrument. Due to the wide coronal temperature coverage achieved with XRT observations, for the first time the XRT can provide complete dynamical evolution information for the XBPs. The study of the spatial and temporal relationship between the solar coronal XBPs and the photospheric and chromospheric magnetic features is an important issue in physics of the Sun. The Hinode/XRT observations 
Table 1. Classification of XBPs.

\begin{tabular}{|c|c|c|c|c|}
\hline XBPs Class & XBPs & $\begin{array}{l}\text { XBPs mean brightness } \\
(\mathrm{DN} / \mathrm{s})\end{array}$ & $\begin{array}{l}\text { XBPs brightness/Background brightness } \\
\text { (where background brightness }=4440 \mathrm{DN} / \mathrm{s} \text { ) }\end{array}$ & Mean ratio \\
\hline \multirow[t]{5}{*}{ Class I } & xbp3 & 157389 & 35.45 & \\
\hline & xbp4 & 288013 & 64.87 & \\
\hline & xbp6 & 145266 & 32.72 & 42.33 \\
\hline & xbp7 & 162340 & 36.56 & \\
\hline & xbp14 & 186745 & 42.06 & \\
\hline \multirow[t]{4}{*}{ Class II } & xbp2 & 53573 & 12.07 & \\
\hline & xbp5 & 58236 & 13.12 & 13.39 \\
\hline & xbp1 & 65445 & 14.74 & \\
\hline & xbp13 & 60567 & 13.64 & \\
\hline \multirow[t]{4}{*}{ Class III } & xbp12 & 36978 & 8.33 & \\
\hline & xbp10 & 26789 & 6.04 & 7.39 \\
\hline & xbp8 & 34676 & 7.81 & \\
\hline & xbp11 & 37873 & 8.53 & \\
\hline
\end{tabular}

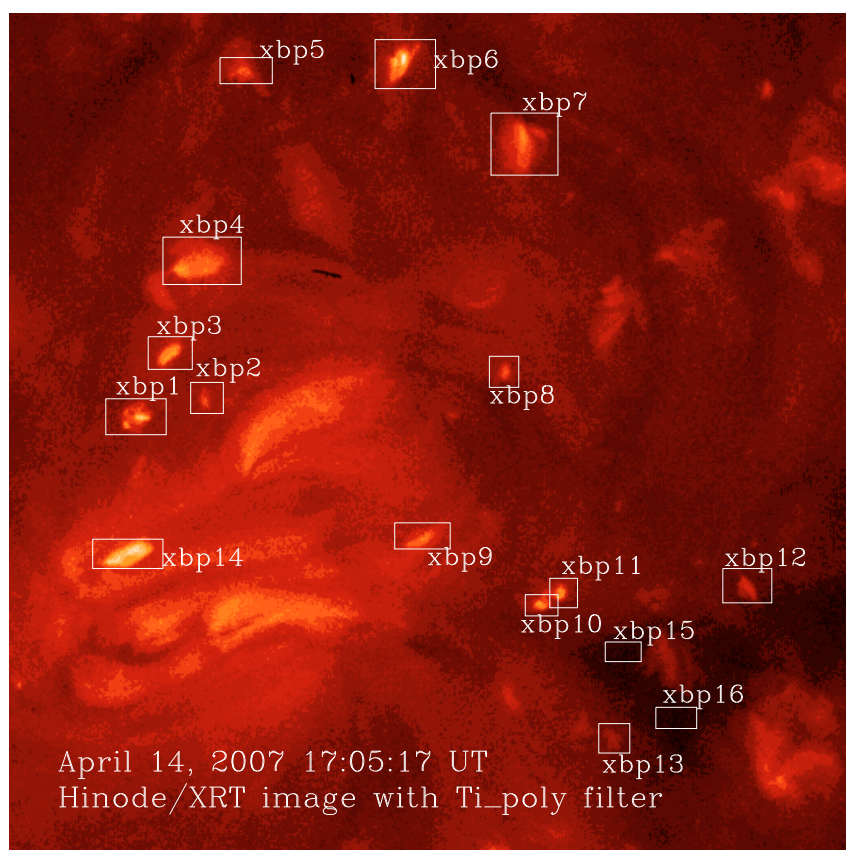

Fig. 1. A sample of an image from the time series obtained by Hinode/XRT on April 14, 2007 at 17:05:17 UT. Where xbp1, $\mathrm{xbp} 2, \ldots \ldots \ldots . . . . \mathrm{xbp} 14$ are the X-ray bright points and $\mathrm{xbp} 15$ and $\mathrm{xbp} 16$ are the background coronal regions selected for the analysis.

provide an opportunity to investigate and understand more deeply the dynamical evolution and nature of the XBP than has been possible to date and to determine their relation to the large-scale magnetic features. Such high resolution observations and investigations would be helpful in understanding the role of oscillations and the nature of the waves associated with XBPs to heat the corona.

In this paper, we report the analysis of 14 XBPs and 2 background coronal regions showing different emission levels selected from $7 \mathrm{~h}$ time sequence observations of soft X-ray images obtained on April 14, 2007. We discuss the results of the periods of intensity oscillations associated with XBPs of different emission levels.

\section{Methods and results}

We use a $7 \mathrm{~h}$ (from 17:00 UT to 24:00 UT) time sequence of soft X-ray images obtained on April 14, 2007 from X-Ray
Telescope (XRT) on-board the Hinode mission. Images were obtained at time cadence of $2 \mathrm{~min}$, but with a short data gap around 18:00 UT. The images were obtained through a single X-ray Ti_poly filter in a quiet region near the center of the solar disk and the image size is $512^{\prime \prime} \times 512^{\prime \prime}$ with a spatial resolution of $1.032^{\prime \prime} /$ pixel. Figure 3 shows a sample of an X-ray image from the time sequence illustrating all the XBPs, which we discuss in more detail below. We have identified and selected 14 XBPs and 2 background coronal regions of the images around 17:00 UT for analysis. We have marked the 14 XBPs on the image in Fig. 1 as $\mathrm{xbp} 1, \mathrm{xbp} 2, \ldots \ldots \ldots . . . . \mathrm{xbp} 14$ and 2 background coronal regions as $\mathrm{xbp} 15$ and $\mathrm{xbp} 16$.

We have used the routine xrt_prep.pro in IDL under SSW to calibrate the images and this routine performs many corrections such as: (i) removal of cosmic-ray hits and streaks (using the subroutine xrt_clean_ro.pro), (ii) calibration of read-out signals, (iii) removal of CCD bias, (iv) calibration for dark current, and (v) normalization of each image for exposure time. On the calibrated images we have placed rectangular boxes covering the selected XBPs and derived the cumulative intensity values of the XBPs by adding all the pixel intensity values. The light curves of all the XBPs and background regions have been derived. The light curves of the background coronal emission show that the fluctuations due to the background coronal emission is very small compared to the intensity variations at the sites of XBPs. However, we assumed that the background coronal emission is more or less constant and subtracted the intensity values of XBPs, point-by-point, from the background coronal intensity values (xbp15 and xbp16) in all the images.

The light curves of 5 XBPs (xbp2, xbp3, xbp4, xbp5, \& $\mathrm{xbp} 12$ ) and one background coronal region (xbp15) are shown in Fig. 3 for comparison of the selected XBPs. The intensity oscillation is seen in all the light curves of the XBPs. There is a short data gap in all the time series around 18:00 UT due to a lack of observations. The light curve of xbp3 is shown in Fig. 3a which indicates a steady increase in brightness with intensity oscillation. Figure $3 \mathrm{~b}$ shows the brightness variation of $\mathrm{xbp} 4$ which is similar to $\mathrm{xbp} 3$ in the intensity variations. Figure $3 \mathrm{c}$ illustrates the light curve of xbp2 exhibiting oscillations with a strong intensity enhancement in the time period from 18:30 UT to 20:30 UT. The light curve of xbp5 is shown in Fig. $3 \mathrm{~d}$ and shows intensity oscillations similar to other XBPs but with a strong intensity enhancement at two locations between 18:0019:00 UT and 21:00-22:00 UT. Similarly the light curve of $\mathrm{xbp} 12$ is presented in Fig. 3e and the intensity oscillations are clearly seen. The light curve of the background coronal region, 

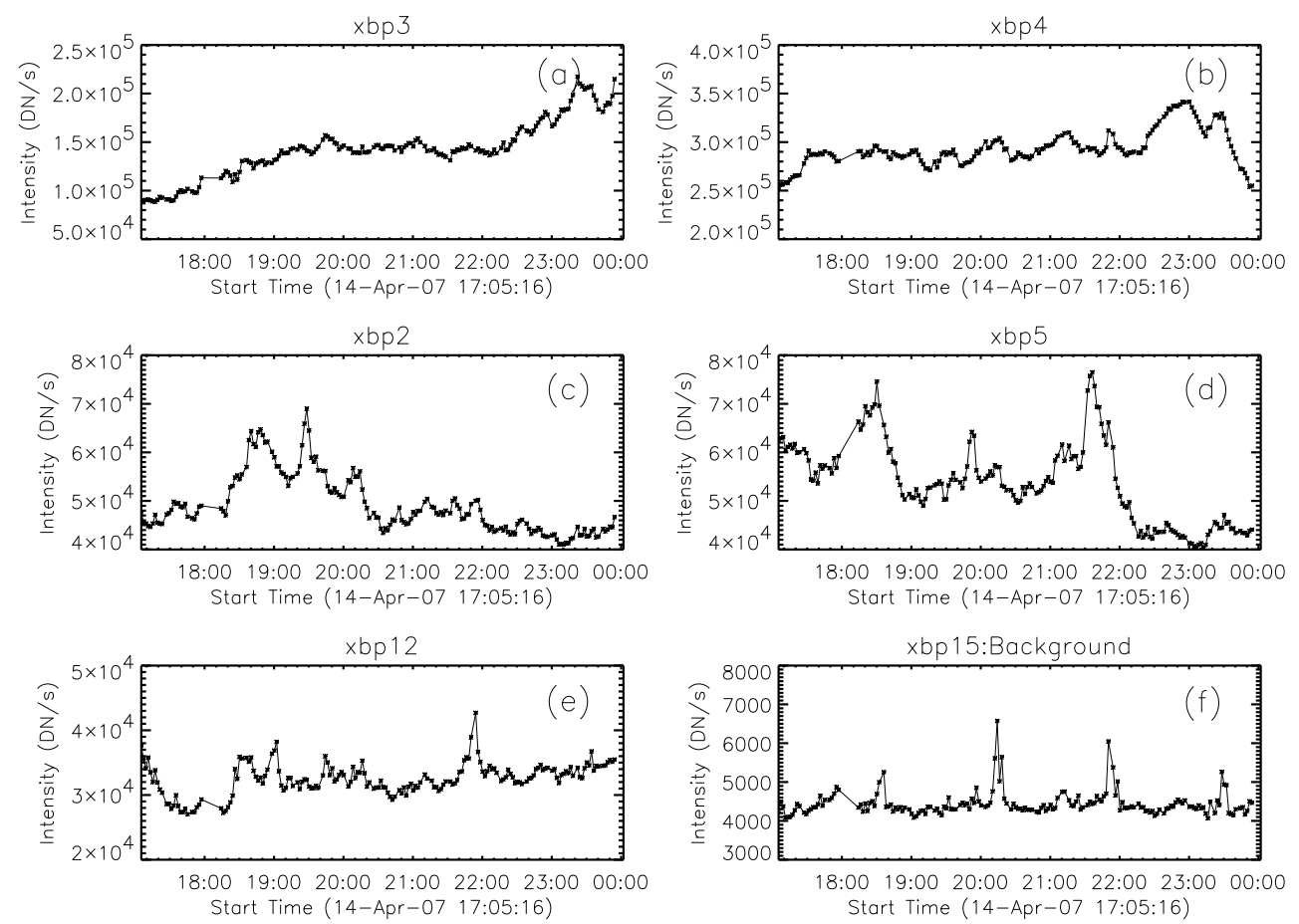

Fig. 2. Time series of XBPs and background coronal region (xbp2, xbp3, xbp4, xbp5, xbp12 \& xbp15 as shown in Fig. 3) of April 14, 2007, where a) xbp3 \& b) xbp4: Class I; c) xbp2 \& d) xbp5: Class II and e) xbp12: Class III and f) xbp15: background coronal region.
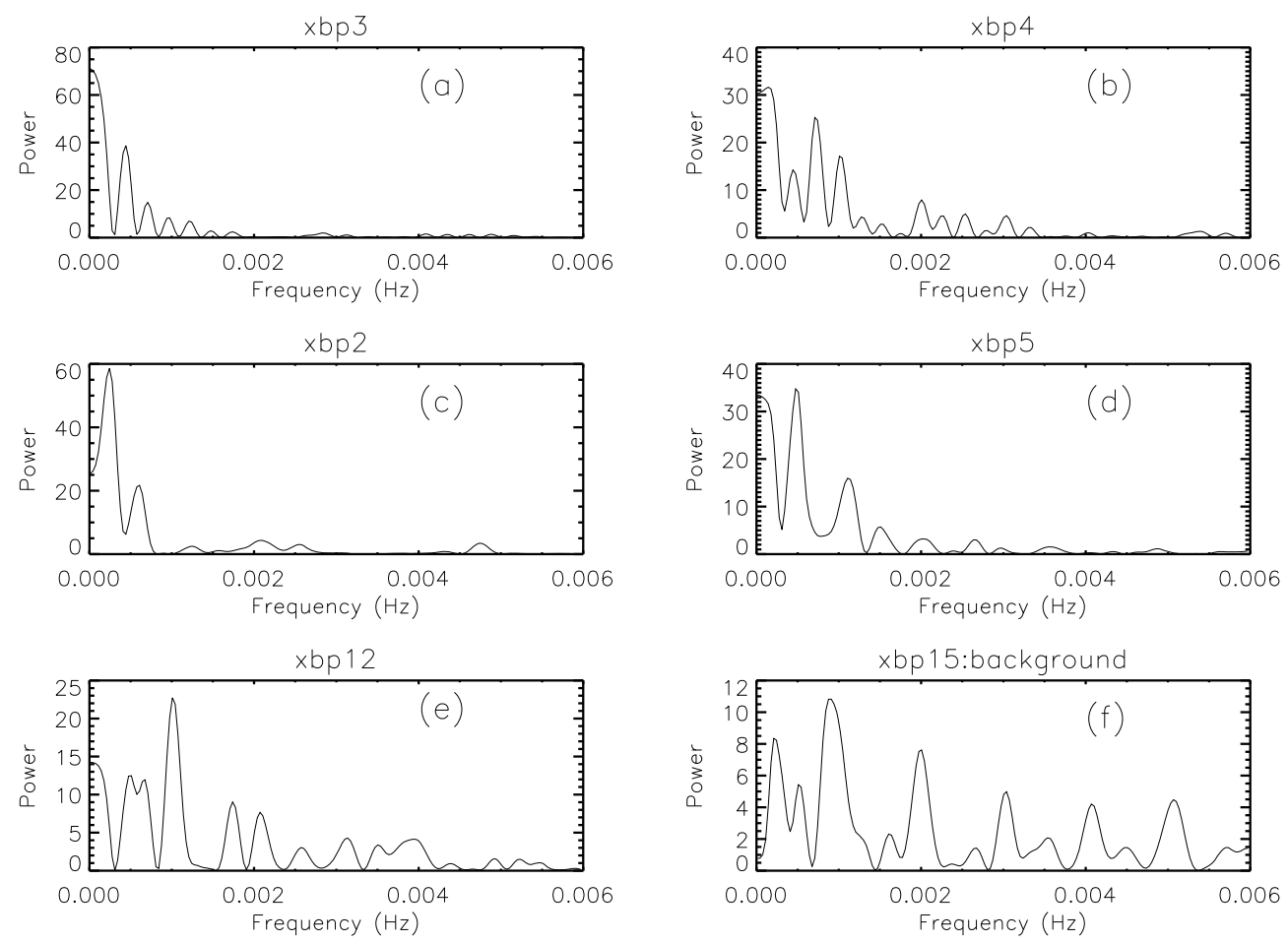

Fig. 3. Power spectra derived for the time series of XBPs and background coronal region (xbp2, xbp3, xbp4, xbp5, xbp12 \& xbp15: background as shown in Fig. 3) of April 14, 2007, where a) xbp3 \& b) xbp4: Class I; (c) xbp2 \& d) xbp5: Class II and e) xbp12: Class III and f) xbp15: background coronal region.

xbp15, is also shown in Fig. 3f which does not show much intensity fluctuation compared to XBPs, except for sharp intensity brightenings about every $80-100 \mathrm{~min}$.

In order to determine the periods of the intensity oscillations, we have carried out a power spectrum analysis of all the XBPs and coronal background regions. The power spectra of 5 XBPs and one background coronal region are shown in Fig. 3.
Figure 3a shows the power spectrum of xbp3 with three significant peaks at the beginning with low frequency values that correspond to the periods of $139 \mathrm{~min}, 37 \mathrm{~min}$ and $24 \mathrm{~min}$. Figure $3 \mathrm{~b}$ illustrates the power spectrum taken for the time series of xbp4 indicating many significant peaks at various frequency values which correspond to the periods of $111 \mathrm{~min}, 37 \mathrm{~min}, 23 \mathrm{~min}$, $17 \mathrm{~min}$ etc. The power spectrum of $\mathrm{xbp} 2$ is shown in Fig. 3c 
and represents two prominent peaks corresponding to the periods of $69 \mathrm{~min}$ and $28 \mathrm{~min}$. The power spectrum of xbp5 is demonstrated in Fig. 3d representing several periods of oscillation, namely $133 \mathrm{~min}, 33 \mathrm{~min}$ and $13 \mathrm{~min}$. Similarly, in Fig. 3e the power spectrum of $x b p 12$ is plotted and shows a variety of periods of $133 \mathrm{~min}, 33 \mathrm{~min}, 17 \mathrm{~min}$ and $9 \mathrm{~min}$. The power spectrum of one of the background regions $(\mathrm{xbp} 15)$ is also shown in Fig. $3 \mathrm{f}$ and has several prominent peaks corresponding to the periods of intensity oscillation as $81 \mathrm{~min}, 31 \mathrm{~min}, 19 \mathrm{~min}$, and $8 \mathrm{~min}$. The power spectra of all the XBPs reveal a wide variety of time scales from a few minutes to hours and some periods of oscillation are similar.

\section{Discussions and conclusions}

The XRT data shows that XBPs tend to produce small and large time scale fluctuations in their intensity and some periods of intensity oscillation are similar in all the XBPs. The periods observed with XRT data ranges from a few minutes to hours and these findings are in good agreement with the results of Strong et al. (1992) derived from the analysis of full-disk images obtained by the Yohkoh/SXT experiment.

Although at first sight the light curves of 14 XBPs seem to be very diverse in their pattern during evolution, we find that they can broadly be grouped into three classes depending on their emission level. The Class I XBPs show a very large intensity enhancement, whereas the Class II XBPs show moderate brightness enhancement and the Class III XBPs show only a marginal intensity enhancement during their dynamical evolution. We have calculated the mean intensity of XBPs from their time series belonging to Class I, Class II and Class III and the background coronal regions and presented the results in Table 1. We determined the mean intensity ratio between the different classes of XBPs and the background coronal emission and the ratios have been listed in Table 1 . The mean background intensity value of $\mathrm{xbp} 15$ and $\mathrm{xbp} 16$ derived over the time sequence is $4440 \mathrm{DN} / \mathrm{s}$. It is seen from the light curves (Fig. 3) and Table 1 that the XBPs will fall into 3 classes depending on the brightness level during their dynamical evolution. Class I XBPs are almost 42 times brighter than the background coronal emission, whereas the Class II and III XBPs are 13 and 7 times brighter than background coronal emission respectively.

We conclude that the analysis of long time series observational data of the XBPs is thus promising and future work will reveal more of the dynamical nature and the physical properties of different oscillations and waves associated with the XBPs. Since the periods of intensity oscillation in all the three cases of XBPs seem to be similar, this can be taken as an evidence that heating mechanisms in the three cases of XBPs are similar. XBPs exhibit a wide variety of time scales ranging from a few minutes to hours in their intensity variations and the periods are almost similar in all the cases of XBPs and thus seems to be independent of the differences in the brightness enhancement. The XBPs are the sites where intense brightness enhancement is seen, and the brightness oscillates with different periods. It suggests that the regions of intense vertical magnetic field strength coincide with regions that are bright, indicating non-radiative heating, irrespective of the sizes of these structures. A comparison between the XBPs and underlying photospheric magnetic features has suggested that the horizontal component of the magnetic field may be playing an important role in driving the brightening of an XBP (Kotoku et al. 2007). Therefore the explanation of the existence of different classes of XBPs with similar periods among all the XBPs may be related to the different strengths of the magnetic field with which they have been associated.

Acknowledgements. Hinode is a Japanese mission developed and launched by ISAS/JAXA, collaborating with NAOJ as a domestic partner, NASA and STFC (UK) as international partners. Scientific operation of the Hinode mission is conducted by the Hinode science team organized at ISAS/JAXA. This team mainly consists of scientists from institutes in the partner countries. Support for the postlaunch operation is provided by JAXA and NAOJ (Japan), STFC (UK), NASA (USA), ESA, and NSC (Norway).

R.K. wishes to thank Tetsuya Watanabe for discussion on several occasions on the topic of XBPs and for his valuable information at the beginning in using the Hinode data. Many thanks to Katharine Reeves who clarified and helped in solving many problems of access to the XRT images and Ed DeLuca for his valuable suggestions and discussion on the XRT observations. The authors are grateful to the referee and to Hardi Peter for many useful comments, suggestions, and constructive remarks which helped considerably in improving the manuscript.

\section{References}

Cram, L. E., \& Damé, L. 1983, ApJ, 272, 355

Curdt, W., \& Heinzel, P. 1998, ApJ, 503, L95

DeLuca, E. E., \& Saar, S. H. 2008, in preparation

Golub, 1., \& Pasachoff, J. M. 1997, The Solar Corona (Cambridge, UK: Cambridge University Press)

Golub, L., Krieger, A. S., Silk, J. K., Timothy, A. F., \& Vaiana, G. S. 1974, ApJ, 189, L93

Golub, L., Krieger, A. S., Harvey, J. W., \& Vaiana, G. S. 1977, Sol. Phys., 53, 311

Habbal, S. R. 1990, in Mechanisms of Chromospheric and Coronal Heating, ed. P. Ulmschneider, E. R. Priest, \& R. Rosner (Springer Verlag), 127

Hara, H., \& Nakakubo-Morimoto, K. 2003, ApJ, 589, 1062

Harvey, K. L. 1996, in Magnetic Reconnection in the Solar Atmosphere, ed. R. D. Bentley \& J. T. Mariska, ASP Conf., Ser., 111, 9

Kariyappa, R. 1994, Sol. Phys., 154, 19

Kariyappa, R. 1996, Sol. Phys., 165, 211

Kariyappa, R. 1999, in 19th NSO/Sac Peak Summer Workshop on High Resolution Solar Physics: Theory, Observations, and Techniques, ASP Conf. Ser., 183,420

Kariyappa, R., \& Pap, J. M. 1996, Sol. Phys., 167, 115

Kariyappa, R., Sivaraman, K. R., \& Anandaram, M. N. 1994, Sol. Phys., 151, 243

Kariyappa, R., Varghese, B. A., \& Curdt, W. 2001, A\&A, 374, 691

Kariyappa, R., Satyanarayanan, A., \& Damé, L. 2005, Bull. Astron. Soc. India, 33,19

Kariyappa, R. 2008, A\&A., under revision

Kariyappa, R., \& DeLuca, E. E. 2008, in preparation

Kotoku, J., Kano, R., Tsuneta, S., et al. 2007, PASJ, 59, S735

Krieger, A. S., Vaiana, G. S., \& Van Speybroeck, L. P. 1971, in Solar Magnetic Fields, ed. R. Howard, IAU Symp., 43, 397

Liu, S. Y. 1974, ApJ, 189, 359

Longcope, D. W., Kankelberg, C. C., Nelson, J. L., \& Pevtsov, A. A. 2001, ApJ, 553,429

Nakakubo, K., \& Hara, H. 1999, Adv. Space Res., 25(9), 1905

Sattarov, I., Pevtsov, A. A., Hojaev, A. S., \& Sherdonov, C. T. 2002, ApJ, 564, 1042

Strong, K. T., Harvey, K., Hirayama, T., et al. 1992, PASJ, 44, L161

Vaiana, G. S., Krieger, A. S., Van Speybroeck, L. P., \& Zehnfennig, T. 1970, Bull. Am. Phys. Soc., 15, 611

Zhang, J., Kundu, M., \& White, S. M. 2001, Sol. Phys., 88, 337 